\title{
Percutaneous Microwave Ablation in the Spleen for Treatment of Hypersplenism in Cirrhosis Patients
}

\author{
XiangWu Jiang ${ }^{1} \cdot$ Fei Gao $^{1} \cdot$ Yan $_{\mathrm{Ma}^{1}} \cdot$ ShuFen Feng $^{1} \cdot$ XueLian Liu $^{1} \cdot$ \\ HongKe Zhou ${ }^{1}$
}

Received: 27 February 2015/ Accepted: 23 May 2015/Published online: 2 June 2015

(c) The Author(s) 2015. This article is published with open access at Springerlink.com

\begin{abstract}
Aim The aim of this study was to estimate the feasibility and therapeutic effectiveness of percutaneous microwave ablation in the treatment of hypersplenism in cirrhosis.

Methods Forty-one cirrhosis patients with hypersplenism were treated with ultrasonography-guided percutaneous microwave ablation between February 2007 and August 2011. Peripheral blood cell counts, portal vein diameter, splenic vein diameter, and blood flow of splenic vein were evaluated before and after the operation, and complications of the treatment were also investigated. All patients were followed up for 24 months.

Results The levels of platelets and white blood cells were increased, while the splenic vein diameter narrowed gradually after the therapy and 24 months later. Moreover, patients received percutaneous microwave ablation had much lower splenic venous flow velocity. The portal vein diameter did not change significantly 6 months after the treatment, although it narrowed gradually within 3 months after the treatment. Furthermore, no complications such as uncontrollable bleeding, splenic abscess, spleen rupture, and damage in surrounding organ happened after the therapy.

Conclusions Graded percutaneous microwave ablation, as a minimally invasive therapy, could damage the spleen, increase the levels of platelets and white blood cells, and reduce portal hypertension effectively without serious
\end{abstract}

XiangWu Jiang and Fei Gao have contributed equally to this work.

XiangWu Jiang

jxr112233@126.com

1 Department of Gastroenterology, The First Affiliated Hospital of Jinan University, Guangzhou 510630, China complications. Percutaneous microwave ablation is an effective, safe, and feasible method for cirrhosis patients with hypersplenism.

Keywords Percutaneous microwave ablation - Cirrhosis · Hypersplenism · Minimally invasive therapy

\section{Introduction}

Cirrhosis is defined as the histological development of regenerative nodules surrounded by fibrous bands in response to chronic liver injury and leads to portal hypertension, hypersplenism, and end-stage liver disease [1]. Hypersplenism, presenting with symptoms of anemia, infection, or bleeding, is a pancytopenia occurring in patients with an enlarged spleen. It is due to large numbers of cells being pooled and destroyed in the spleen's reticuloendothelial system, and hemodilution because of an increased plasma volume.

Open splenectomy (OS) has been performed for hypersplenism, but OS is excessively invasive in terms of blood loss and wound pain. Splenectomy is associated with high rates of morbidity and mortality. Catheter-based arterial embolization has gradually become more popular, but some severe complications have been reported, which limit its wide use [2]. Microwave ablation has been reported to be a safe and effective technique for the management of hypersplenism in patients with liver cirrhosis [3]. The aim of this article is to give an overview of the latest development in percutaneous microwave ablation for hypersplenism secondary to liver cirrhosis and portal hypertension, as well as to evaluate the feasibility and safety of percutaneous microwave ablation in hypersplenism. 


\section{Materials and Methods}

\section{Patients}

In this prospective study, 41 cases of cirrhosis patients with hypersplenism were admitted between February 2007 and August 2011; all of the patients had splenomegaly, with decreased red blood cells, white blood cells, and platelets separately or simultaneously. Patients were excluded if they suffered from severe heart and brain disease; had severe coagulopathy; had gastrointestinal bleeding, severe jaundice, and massive ascites of hepatic encephalopathy recently; and had hepatocellular carcinoma and portal vein or hepatic vein thrombosis.

Of the 41 patients, 30 were male and 11 were female, and the mean age of the patients was 47 . Thirty-eight cases of cirrhosis patients were caused by hepatitis B, two were caused by hepatitis $\mathrm{C}$, and one was caused by primary biliary cirrhosis. Preoperative assessment of liver function was also carried out, and 27 were Child-Pugh class A, 12 were Child-Pugh class B, and two were Child-Pugh class C. Four cases had mild degree of splenomegaly $[<5 \mathrm{~cm}$ below the left costal margin (LCM)], 32 cases had moderate degree of splenomegaly $(5-8 \mathrm{~cm}$ below LCM and maximum cephalocaudal diameter being 11-20 cm), and five cases had severe degree of splenomegaly $(>8 \mathrm{~cm}$ below LCM and maximum cephalocaudal diameter being $>20 \mathrm{~cm}$ ). The average preoperative white blood counts were $2.45 \pm 0.81 \times 109 / \mathrm{L}$, the average preoperative red blood counts were $3.44 \pm 0.71 \times 10^{12} / \mathrm{L}$, and the average preoperative platelet counts were $48.54 \pm 18.92 \times 10^{9} / \mathrm{L}$. All of the patients were carried out percutaneous microwave ablation twice and followed up for at least 24 months.

\section{Laboratory Examination and Preparation Before Ablation}

Complete blood count tests were performed before the procedure, CT scans were used to measure the splenic volume, and abdominal ultrasound was used to evaluate the portal vein diameter, splenic vein diameter, and the blood flow velocity. All patients were admitted to the ward, received percutaneous microwave ablation voluntarily, and signed informed consent after fasting for $6 \mathrm{~h}$ before MW ablation.

\section{Percutaneous Microwave Ablation Procedure}

A detailed protocol including the operation method, an appropriate approach, placement of the applicators, power output, ablation time, and emission times was worked out for each patient on an individual basis before treatment. ECO-100C microwave treatment system (60-70 W) from Nanjing ECO Microwave System Engineering Co., Ltd. was used in the ablation procedure. For percutaneous microwave splenic ablation, pethidine hydrochloride was injected intramuscularly about $10 \mathrm{~min}$ before surgery for sedative. After local anesthesia using lidocaine, the spleen was accessed percutaneously via the left subcostal approach under ultrasound guidance. With the MW emitting for $15 \mathrm{~min}$, the bleeding stopped spontaneously. After emission, the applicator was pulled back for about $3 \mathrm{~cm}$. MW was reemitted until the ablation zone was $<2 \mathrm{~cm}$ from the splenic surface. Two to three overlapping areas from middle to inferior part of the spleen were thus ablated with one insertion of applicators. Ablation range can be estimated and ablation volume percentage can be calculated during the operation. Percutaneous fractionated microwave ablation was adopted herein. We chose 4-5 puncture points each time and expected that about one-third of the whole spleen was ablated. The second ablation was arranged 1 week later.

\section{Follow-up}

Complete blood count tests (the white blood cell count, platelet count, and red blood cell count) were valuated 3 days, 7 days, 1 month, 3 months, 6 months, 12 months, 18 months, and 24 months after microwave ablation among all 41 patients. The ablation area, portal vein, splenic vein diameter, and blood flow velocity were also measured by abdominal ultrasound for each patient 3 days, 7 days, 1 month, 3 months, 6 months, 12 months, 18 months, and 24 months after microwave ablation. What is more, abdominal CT scans were used to investigate changes in the ablation zone and calculate the ablation volumes 1 month after the procedure. Postoperative follow-up also included whether there were complications, such as upper gastrointestinal bleeding, liver failure, or death.

\section{Ethics Statement}

Approvals from the First Affiliated Hospital of Jinan University Institute Research Ethics Committee were obtained, and written informed consent was provided by each human subject.

\section{Statistical Analysis}

The statistical analyses were performed using the SPSS 18.0 software package. Data are presented as the mean \pm SEM. Two-tailed Student's $t$ test was used for the 
comparisons of two independent groups. A $p$ value of $<0.05$ was considered to be statistically significant.

\section{Results}

All treatments were technically successful. Microwave ablations were induced by using different power outputs $(60-70 \mathrm{~W})$. The average ablation times were $8.8 \pm 1.3$, and the mean ablation time was $71.4 \pm 12.1 \mathrm{~min}$.

\section{The Ablation Zones in Ultrasound Images}

In the process of microwave ablation, the hyperechoic regions closely related to the ablation zones were microbubbles formed of spleen tissue vaporization when heated up, and could spread outward gradually (Fig. 1a). The lesion after ablation could be defined into three areas: charred area around the needle track, central necrotic area, and reaction zone around. Then, the needle tract coagulated and showed narrow hyperechoic bands in the ultrasound images, while ablation area surrounding the needle tract showed a homogeneous hyperechoic appearance 7 days after the operation. Moreover, with postoperative spleen fibrosis and volume reduction, the ablation area displayed uneven streak echoes (Fig. 1b) and hyperechoic punctate calcifications (Fig. 1c).

\section{The Ablation Zones in Abdominal CT Scan Images}

As shown in Fig. 2a, b, abdominal CT scans demonstrated a complete splenic capsule and an uneven hypodensity in ablation areas of the spleen, which exhibited no enhancement at arterial phase. The CT scan data were imported into commercial Software Mimics (Materialise NV, Belgium), and a 3D graphic model of each spleen was developed to calculate the volume proportion of ablation
(Fig. 2c, d). In this study, we found the mean volume proportion of ablation was $47.2 \pm 7.0 \%$.

\section{Changes in Complete Blood Count Tests}

The mean WBC count before microwave ablation was $2.45 \pm 0.81 \times 10^{9}$ cells/L; it peaked at 3 days $(5.39 \pm$ $2.06 \times 10^{9}$ cells $\left./ \mathrm{L}\right)$ after ablation and then decreased gradually over time. Statistical analysis showed a significant increase in WBC count 7 days, 1 month, 3 months, 6 months, 1 year, 1.5 years, and 2 years after microwave ablation in all patients ( $p<0.05$; Fig. 3a).

The mean PLT count before microwave ablation was $48.54 \pm 18.92 \times 10^{9}$ cells/L, and it decreased to $43.69 \pm$ $16.39 \times 10^{9}$ cells/L 3 days after treatment. However, it increased hereafter and reached a peak level at 30 days after treatment. The mean peak PLT count was $100.92 \pm$ $25.51 \times 10^{9}$ cells/L. Then, it decreased gradually over time. There were significant differences between PLT counts before ablation and those at 1 month, 3 months, 1 year, 1.5 years, and 2 years after microwave ablation in all groups ( $p<0.05$; Fig. 3b).

\section{Portal Vein Diameter, Splenic Vein Diameter, and Blood Flow of Splenic Vein Before and After the Operation}

As shown in Fig. 4a, the mean diameter of the portal vein was $14.62 \pm 1.56 \mathrm{~mm}$ before the operation and was significantly narrowed 3 months $(13.26 \pm 1.36 \mathrm{~mm})$ after the therapy compared with 7 days $(14.33 \pm 0.84 \mathrm{~mm})$ or 1 month $(14.02 \pm 1.16 \mathrm{~mm})$ after the operation $(p<$ $0.05)$, but was not significantly varied 6 months $(14.57 \pm$ $1.07 \mathrm{~mm}$ ) after the therapy.

The splenic vein diameter was $10.51 \pm 1.37 \mathrm{~mm}$ before the operation and was significantly reduced at 7 days (9.24 $\pm 1.34 \mathrm{~mm}), 1$ month $(9.11 \pm 1.06 \mathrm{~mm}), 3$ months $(8.97 \pm 1.28 \mathrm{~mm}), 6$ months $(8.86 \pm 1.00 \mathrm{~mm}), 1$ year
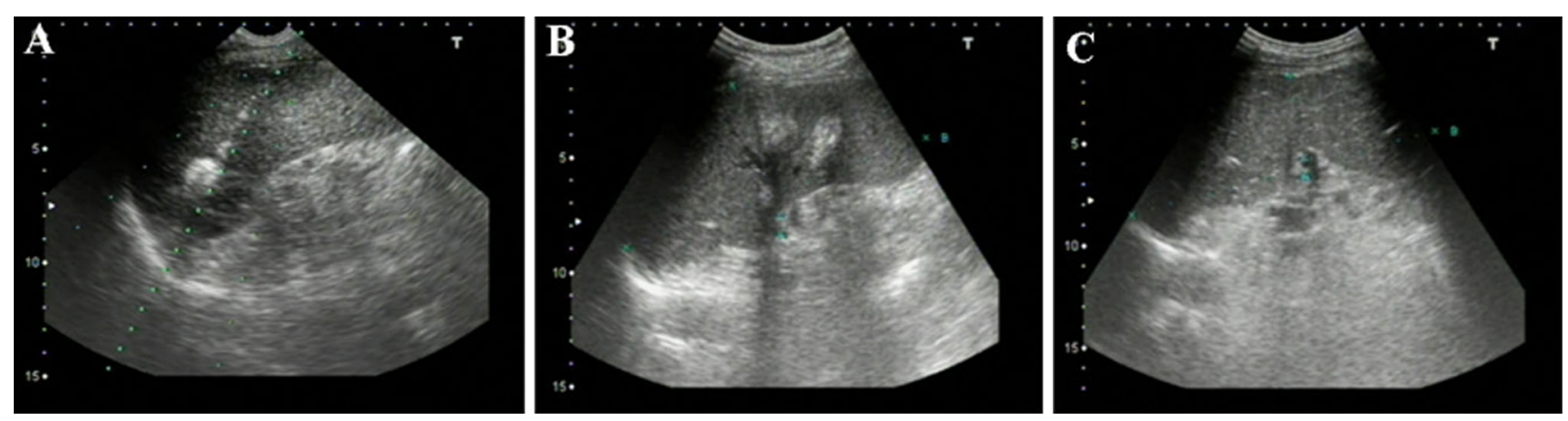

Fig. 1 Ablation zones in ultrasound images. a Ultrasound image during ablation; b ultrasound image of 1 months after the operation; c ultrasound image of 2 years after ablation 

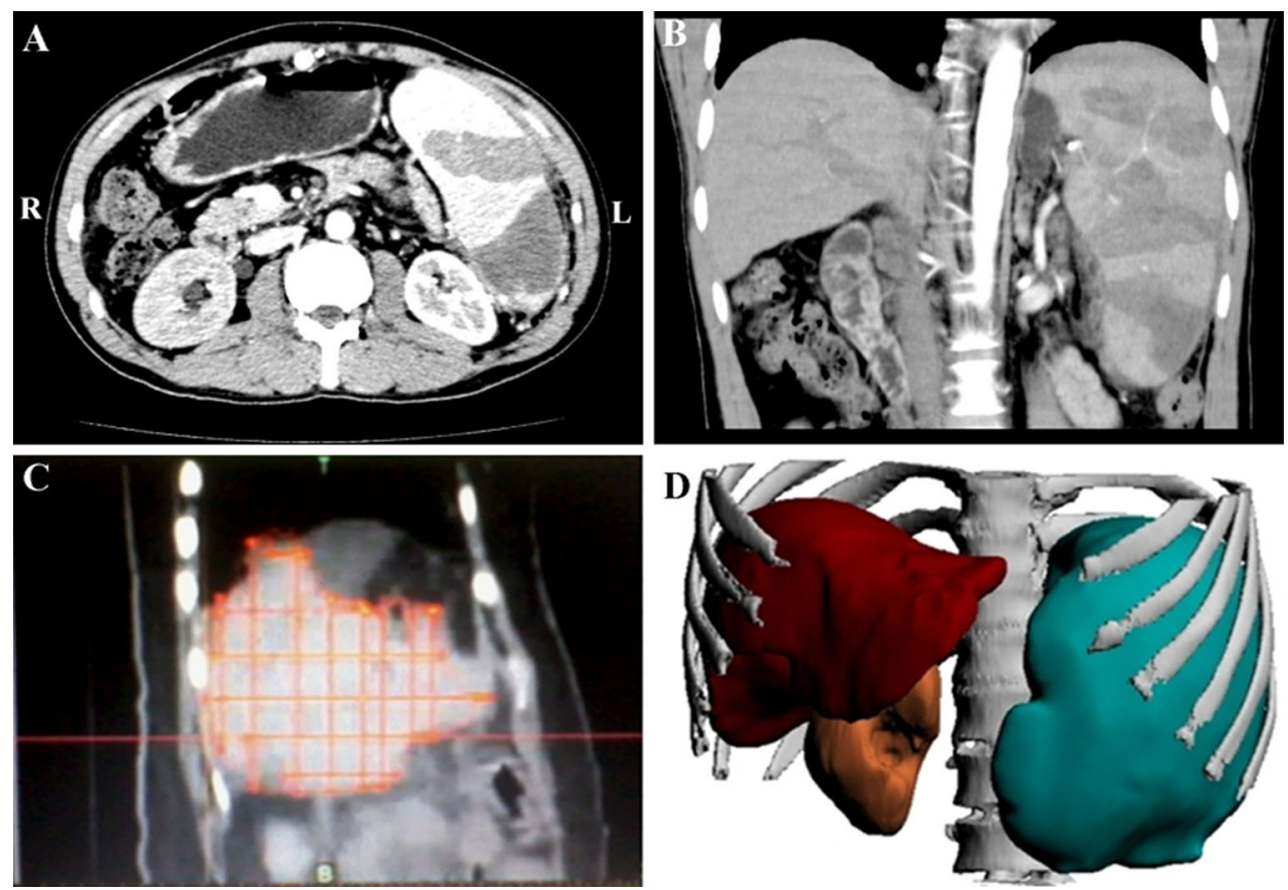

Fig. 2 Ablation zones in abdominal CT scan images. a Arterial-phase image after ablation by CT scan at cross section; $\mathbf{b}$ arterial-phase image after ablation by CT scan at coronal plane; c-d 3D graphic model of spleen
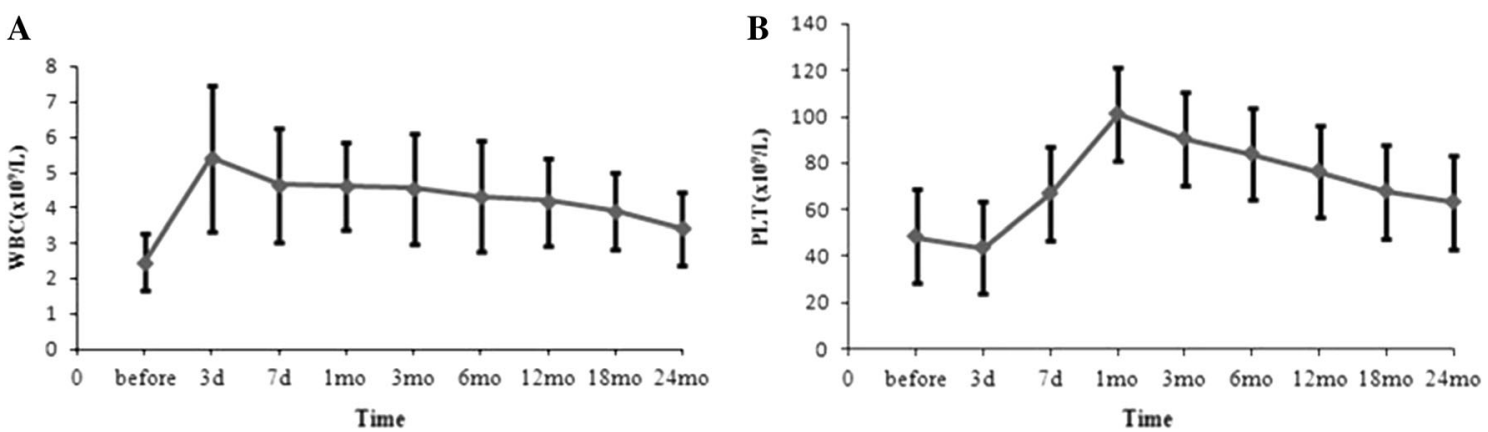

Fig. 3 Changes in complete blood count tests. a Changes in WBC count; b changes in PLT count

$(8.80 \pm 0.98 \mathrm{~mm}), \quad 1.5$ years $(8.89 \pm 1.07 \mathrm{~mm})$, and 2 years $(9.06 \pm 1.15 \mathrm{~mm})$ after ablation (Fig. $4 \mathrm{~b})$.

Moreover, as shown in Fig. 4c, we also detected the blood flow of splenic vein and found decreased splenic blood flow at 7 days, 3 months, 6 months, 12 months, 18 months, and 24 months after ablation.

\section{Complications of the Treatment}

Complications happened after the treatment included fever, transient hemoglobinuria, left upper abdomen or left shoulder pain, and a small amount of pleural effusion. There were no severe complications related to ablation, for example, uncontrollable bleeding, abscesses or rupture of the spleen, burned skin in treatment area, skin bruising, damage to surrounding organs such as the pancreas, stomach, colon, and left kidney, and even death.

Of the 41 patients, all of them developed different degrees of fever and suffered from transient hemoglobinuria which could recover in $24 \mathrm{~h}$. Twenty-six (63.4\%) suffered from slight left upper abdomen or left shoulder pain which could relieve in 2-5 days. Eleven (26.8 \%) developed a small amount of pleural effusion 3-5 days after ablation which could be self-absorbed 1 week later. Three $(7.3 \%)$ suffered from vomiting and abdominal distension which disappeared after symptomatic treatment. Two developed delayed abdominal bleeding that stopped after athletic bandaged.

There were no significant differences in serum lipase, amylase, and renal function before and after ablation. 

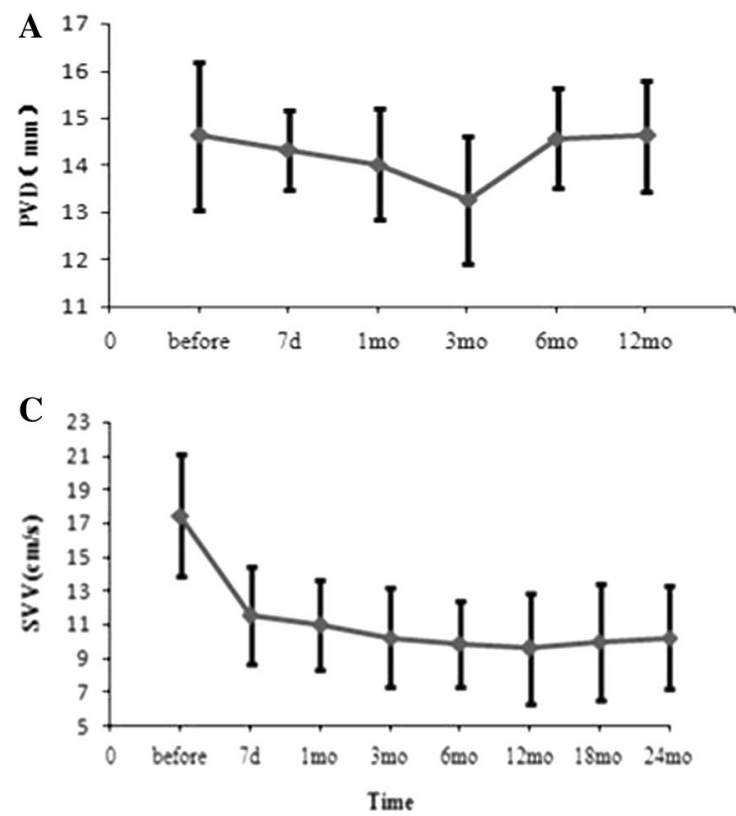

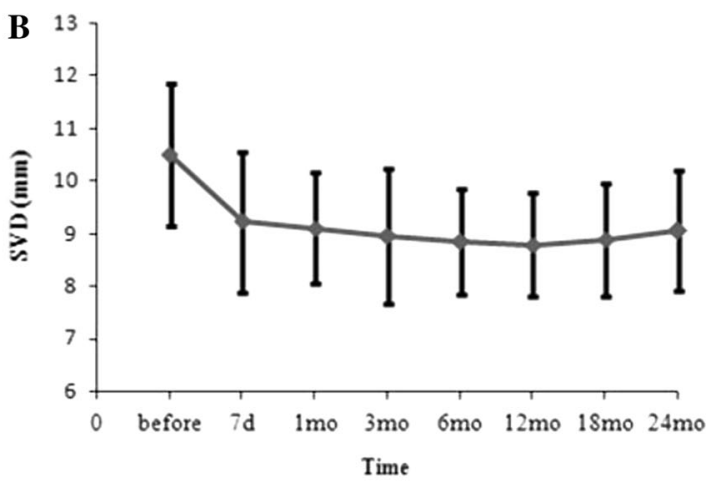

Fig. 4 Portal vein diameter, splenic vein diameter, and blood flow of splenic vein before and after the operation. a Portal vein diameter before and after the operation; b splenic vein diameter before and after the operation; c blood flow of splenic vein before and after the operation

\section{Follow-up}

Patients were followed up for 2 years. Six developed bleeding esophageal varices, one developed hepatic encephalopathy, one died from primary liver cancer, and one died from multi-organ failure. Other 32 patients had lived well and had an overall high quality of life after ablation.

\section{Discussion}

Cirrhosis is an advanced stage of liver fibrosis and most commonly caused by alcoholism, hepatitis B and hepatitis $\mathrm{C}$, fatty liver disease, as well as many other possible causes. Hypersplenism is a disorder in which the spleen becomes increasingly active and then rapidly and prematurely destroys blood cells. Moreover, hypersplenism is a known complication of portal hypertension secondary to cirrhosis of the liver.

Most individuals with secondary hypersplenism require therapy to prevent further destruction of blood cells and possible spleen enlargement, but so far, no universally accepted therapy has been established for hypersplenism. For the therapy of hypersplenism in cirrhosis patients, laparoscopic splenectomy was reported as a technically feasible, safe, and effective procedure for hypersplenism secondary to cirrhosis and portal hypertension and contributed to decreased blood loss, a shorter hospital stay, and less impairment of the liver function compared with traditional open splenectomy [2]. It was reported that partial splenic embolization should be attempted prior to consideration of removal of the spleen. Partial splenic embolization is a simple, rapid procedure that is easily performed under local anesthesia, and it allows preservation of adequate splenic tissue to safeguard against overwhelming infection [4, 5].

Percutaneous microwave ablation was first used as an adjunct to liver biopsy in 1986, but it has since been used for hepatic tumor ablation, nonmetastatic lung tumors, renal carcinomas, secondary splenomegaly, and hypersplenism. Microwave ablation is a special case of dielectric heating, where the dielectric material is tissue. Dielectric heating occurs when an alternating electromagnetic (EM) field is applied to an imperfect dielectric material. In tissue, heating occurs because the EM field forces water molecules in the tissue to oscillate. The bound water molecules tend to oscillate out of phase with the applied fields, so some of the EM energy is absorbed and converted to heat. The best EM absorbers contain a high percentage of water, while less heating occurs in tissues with low water content. A common measure used to describe how efficiently a material will absorb EM energy is effective conductivity $(\sigma)$. At microwave frequencies (typically $915 \mathrm{MHz}$ or $2.45 \mathrm{GHz}$ for ablative technologies), heating is more efficient in materials with a high conductivity [3, 6, 7]. Microwave ablation is an ablative technique similar to radiofrequency or cryosurgical ablation; however, microwave ablation has some potential hypothetical advantages over radiofrequency or cryosurgical ablation. In this study, we found percutaneous microwave ablation under 
the guidance of an ultrasound for hypersplenism increased platelet and white blood cell counts. It also helped to maintain splenic immunologic function and even improved splenic immunologic function in a short term. The complications happened after the treatment mainly included fever, transient hemoglobinuria, left upper abdomen or left shoulder pain, and a small amount of pleural effusion; however, there were no severe complications related to ablation. The above-mentioned results indicate that ultrasound-guided percutaneous microwave ablation is a feasible, safe, and efficient method for the treatment of secondary splenomegaly and hypersplenism in cirrhosis patients.

During the operation, how to reduce the risk of bleeding while ablate more splenic volume safely is of the most importance. In our operation, in order to reduce bleeding, we put the needle quickly and accurately into and out of the spleen after the area under skin was anesthetized, the needle tract and spleen capsule should be cauterized by withdrawing the needle, and local anesthetic was used instead of intravenous anesthesia to avoid splenic capsule tearing and diaphragmatic injury induced by ablation needle shaking or abdominal breathing. We carried out the second ablation by withdrawing the needle to the area without overlapping the first ablation area. Moreover, when finished the ablation, we used ultrasound to detect bleeding by showing whether there were any peritoneal fluid areas. Puncture sites were athletic bandaged after the ablation, patients should stay in bed for $24 \mathrm{~h}$, and the blood pressure, pulse, temperature, and other vital signs should be closely monitored. We ablated twice for each patient in order to increase ablation volume while reducing complications and ablated one-third of the splenic volume each time, and the interval from the second time to the first ablation was about 1 week.

However, there are still several limitations of microwave ablation. For example, the best combination of ablation site, ablation power, and ablation time needs to be further studied. The best ablation volume is also inconclusive, and we will explore the relationship between ablation volume, treatment effects, and the complications to maximize the effect of ablation in the future study. What is more, whether liver function, hypersplenism severity, age, and gender are associated with ablation effect needs discussion by summarizing the characteristics of cases. In addition, how long the microwave ablation efficacy can be maintained needs longer clinical follow-up studies.

In the future study, we will collect data of other treatment methods and compare the efficacies and the risks between groups. An evidence-based medical research of larger samples, multicenter, and unified standards needs to be carried out. Furthermore, indications and contraindications of microwave ablation in the treatment of hypersplenism should be standardized, and its efficacy, complications, and recurrence rate still need a longer follow-up.

Conflict of interest No potential conflicts of interest were disclosed.

Open Access This article is distributed under the terms of the Creative Commons Attribution-NonCommercial 4.0 International License (http://creativecommons.org/licenses/by-nc/4.0/), which permits any noncommercial use, distribution, and reproduction in any medium, provided you give appropriate credit to the original author(s) and the source, provide a link to the Creative Commons license, and indicate if changes were made.

\section{References}

1. Schuppan D, Afdhal NH. Liver cirrhosis. Lancet. 2008;371: 838-851.

2. Zhan XL, Yun JI, Wang YD. Laparoscopic splenectomy for hypersplenism secondary to liver cirrhosis and portal hypertension. World J Gastroenterol. 2014;20:5794-5800.

3. Liang P, Gao Y, Zhang H, et al. Microwave ablation in the spleen for treatment of secondary hypersplenism: a preliminary study. Am J Roentgenol. 2011;196:692-696.

4. Moreno A, Bárcena R, Blázquez $\mathrm{J}$, et al. Partial splenic embolization for the treatment of hypersplenism in cirrhotic $\mathrm{HIV} / \mathrm{HCV}$ patients prior to pegylated interferon and ribavirin. Antivir Ther. 2004;9:1027-1030.

5. Amin MA, El-gendy MM, Dawoud IE, et al. Partial splenic embolization versus splenectomy for the management of hypersplenism in cirrhotic patients. World J Surg. 2009;33:1702-1710.

6. Tabuse Y, Tabuse K, Mori K, et al. Percutaneous microwave tissue coagulation in liver biopsy: experimental and clinical studies. Nippon Geka Hokan. 1986;55:381-392.

7. Brace CL. Radiofrequency and microwave ablation of the liver, lung, kidney, and bone: what are the differences? Curr Probl Diagn Radiol. 2009;38:135-143. 